\title{
Intertester Reliability of Active and Passive Ankle Joint Position Sense Testing
}

\author{
By: Joseph E. Szczerba, Julie N. Bernier, David H. Perrin, and Bruce M. Gansneder
}

Szczerba, J.E., Bernier, J.N., Perrin, D.H., \& Gansneder, B.M. (1995). Intertester reliability of active and passive ankle joint position sense testing. Journal of Sport Rehabilitation, 4: 282-291.

\section{***Note: Figures may be missing from this format of the document}

\begin{abstract}
:
The purpose of this study was to estimate intertester reliability of active and passive ankle joint position sense measurements in uninjured subjects. Subjects were 10 males and 10 females. Active and passive ankle joint position sense was assessed by two testers. Each subject was positioned supine on a modified examination table with his or her ankle placed in $25^{\circ}$ of plantar flexion. Joint position sense (JPS) measurements, on two separate occasions, were recorded in degrees of error from four predetermined test positions. Test order was counterbalanced according to mode (active/passive) and test position. Two trials were performed for each sequence and the average of the two was recorded for analysis. The results revealed that both the active and passive JPS protocols yielded poor to moderate intertester reliability. It was concluded that further research is needed to develop reliable protocols for testing joint position sense of the ankle joint.

\section{Article:}

The ankle is the most frequently injured joint in the human body, with its lateral ligamentous complex sustaining approximately $85 \%$ of the sprains occurring within this structure $(3,10,13$, 14). Ankle joint injury can impose a significant disability to athletes of all sports, since athletes frequently experience repeated lateral ankle sprains following their initial trauma, which often renders them functionally unstable $(6,7)$.
\end{abstract}

The high incidence of lateral ankle sprains and the high rate of reinjury have prompted many researchers to examine the possible causes for this chronic instability. Three causes of instability have been suggested: a decrease in muscular strength of the ankle evertors $(21,28)$, an increase in lateral ligamentous laxity (21), and proprioceptive deficits resulting from a disruption in the integrity of the joint mechanoreceptors $(8,9,11,21,22,28)$. The theory regarding proprioceptive deficits has recently captured the attention of many clinicians and researchers $(2,14,15,19,23)$.

Proprioception has been described by Lephart et al. as "a specialized variation of the sensory modality of touch [which] encompasses the sensations of joint movement (kinesthesia) and joint position (joint position sense)" (23). This afferent sensory feedback mechanism depends upon various peripheral mechanoreceptors found in the surrounding skin, muscles, tendons, ligaments, and joints (12). These mechanoreceptors respond to mechanical deformation by initiating action

\footnotetext{
* Joseph E. Szczerba, Jr., is a doctoral student in Sports Medicine, University of Virginia, Charlottesville, VA 22903. Julie N. Bernier is with the Department of HPER, Plymouth State College, Plymouth, NH 03264. David H. Perrin and Bruce M. Gansneder are with the Curry School of Education, University of Virginia, Charlottesville, VA 22903.
} 
potentials that are conducted to and processed by the central nervous system (12). As a result, an individual is able to perceive both movement (kinesthesia) and position (joint position sense) of a joint.

Proprioception has traditionally been assessed two ways. Kinesthesia, the perception of joint movement, has been measured via the threshold to detection of passive motion (TTDPM) method $(9,21,23)$. On the other hand, joint position sense has been documented as an individual's ability to reproduce a predetermined joint angle either actively or passively $(2,11$, $13,14,17,19,20)$. Both types of measurement are typically conducted in the open kinetic chain, yet no standardization of methods or equipment has been formally adopted. Joint position sense testing instruments have ranged from the mos basic standard goniometer $(1,11)$ to the more modern isokinetic dynamometers equipped with their own internal electrogoniometers, such as the Orthotron II (Cybex, Division of Lumex, Ronkonkoma, NY) (20), the Cybex II+ (Cybex, Division of Lumex, Ronkonkoma, NY) $(13,17)$, and the Kin-Com II (Chattecx Corporation, Division of Chattanooga, Hixson, TN) $(2,14)$. Still others have specifically designed their own equipment for measuring joint position sense (23).

Unfortunately, many conclusions regarding ankle joint proprioception, as measured via joint position sense, have been drawn without first establishing the reliability of measurements. It is of the utmost importance, in sports medicine as well as in any discipline, that the reliability of measurements be established before any conclusions based upon these measurements can be deemed valid (4). To the best of our knowledge, the reliability of assessing ankle joint position sense has yet to be determined. Therefore, the purpose of this study was to examine the intertester reliability for assessing active and passive ankle joint position sense measurements in uninjured subjects using the Kin-Com II isokinetic dynamometer.

\section{METHODS}

Subjects

Twenty healthy subjects (10 females and 10 males, age $=22.8 \pm 3.7$ years, height $=169.7 \pm 9.0$ $\mathrm{cm}$, weight $=67.2 \pm 11.8 \mathrm{~kg}$ ) volunteered to participate in this study. None of the subjects had a history of ankle sprains, and all were free from lower extremity injury at the time of the study. Prior to participating, each subject read and signed a human consent form approved by a University Committee for the Protection of Human Subjects. Subjects were randomly assigned to one of two testers for initial testing and subsequently returned to the sports medicine research lab within 9 days to be reevaluated by the other tester.

\section{Test Apparatus and Procedures}

The Kinetic Communicator II (Kin-Com II) isokinetic dynamometer was used to assess joint position sense. A constant velocity of $5 \%$ s was selected as the testing speed. The subject was positioned supine on a modified examination table with the lower leg parallel to the floor, resting on a 10-in. high rectangular support (25 in. wide and 14 in. long) (Figure 1). This setup enabled proper placement of the subject's bare foot into the Kin-Com II ankle inversion/eversion apparatus with the ankle plantar flexed $25^{\circ}$. The ankle to be tested was randomly selected. The heel support of the apparatus was lined with 1/2 in. foam, and the subject's midfoot and forefoot were wrapped approximately three times with $1 / 8$ in. Elastifoam wrap (low-density elastic foam) 
to decrease external sensory stimulation. After the subject's foot was properly aligned with the axis of the isokinetic dynamometer according to manufacturer's guidelines, stabilization was provided by placing Velcro straps around the proximal tibiofibular joint and forefoot (Figure 1). Subjects were required to close their eyes during the testing procedures to eliminate visual feedback.

Active and passive joint repositioning occurred at the following four test positions: $10^{\circ}$ eversion, $0^{\circ}$ subtalar neutral, $15^{\circ}$ inversion, and $5^{\circ}$ from maximum inversion. Subtalar neutral served as the $0^{\circ}$ neutral position. This position was individually determined by palpating for equal distribution of the head of the talus bone medially and laterally while passively inverting and everting the foot (16). The $5^{\circ}$ from maximum inversion test position was established by having the subject actively invert the foot maximally. This position was visually displayed in degrees by the internal goniometer of the Kin-Com II. Testing order was counterbalanced according to mode (active/passive) and test position $\left(10^{\circ}\right.$ eversion $/ 0^{\circ}$ subtalar neutral, $15^{\circ}$ inversion $/ 5^{\circ}$ from maximum inversion). The preselected testing order and side of body tested during the initial evaluation were replicated during the second evaluation.

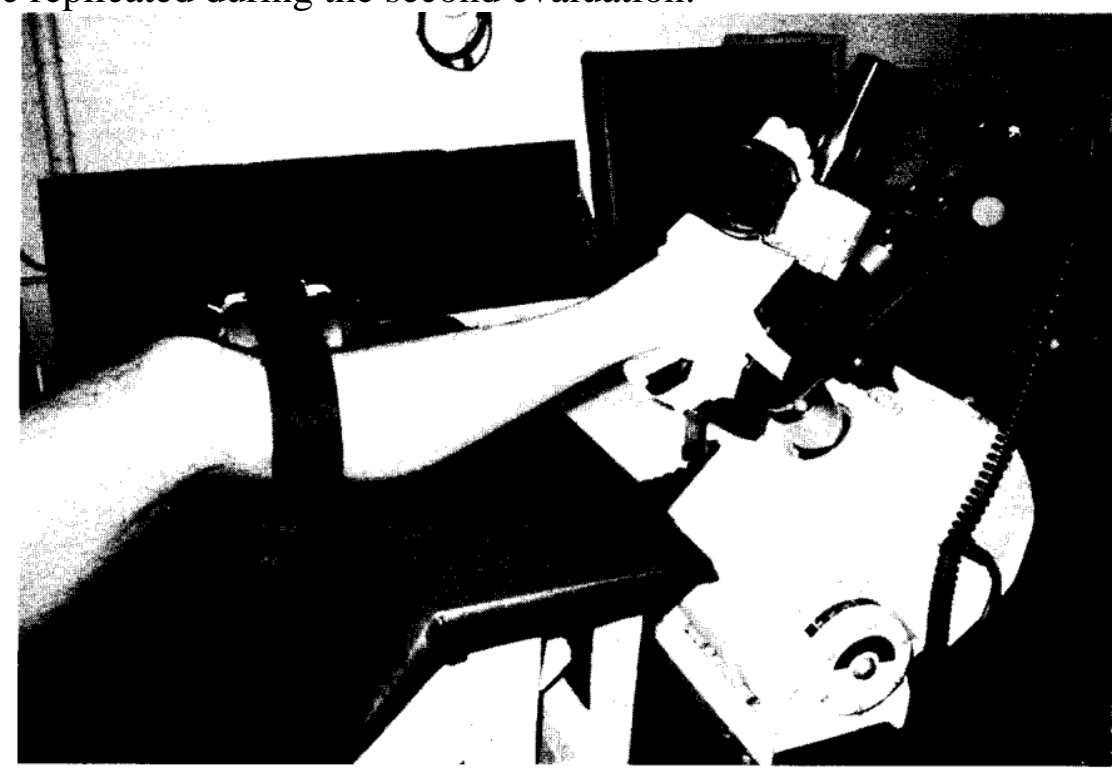

igure 1 - Positioning of subject on the Kin-Com II isokinetic dynamometer fo Inkle joint position sense testing.

Prior to being tested, each subject received a practice session followed by a 30-s rest period. The testing began by the investigator passively moving the subject's foot through its entire inversion and eversion range of motion. The foot was then passively moved into the preselected test position where it was held for $15 \mathrm{~s}$. Once at the test position, the subject concentrated on the test angle. The foot was then passively moved back to the starting position, which was end range of maximal inversion for the $0^{\circ}$ subtalar neutral and $10^{\circ}$ eversion test positions, and end range of maximal eversion for the $15^{\circ}$ inversion and $5^{\circ}$ from maximum inversion test positions. Once the starting position had been reached, the investigator proceeded to passively move the subject's foot back in the direction of the test position. When the subjects felt that their foot position replicated that of the testing angle they performed a quick contraction of the foot in the direction opposite the passive motion. This contraction was then recorded as a spike on the forcevelocity curve output on the Kin-Com II computer screen. From this spike on the curve, the 
investigator could then extrapolate the initial angle of movement that corresponded to the subject's repositioned joint angle. Joint position sense measurements were recorded in degrees of error from the test position. Two trials were performed for each sequence. The absolute values of each error score were then averaged together and recorded for analysis. Active joint position sense testing occurred under an identical protocol except that the subject actively moved his or her foot to replicate the test position after being moved to the start position.

\section{Statistical Analysis}

The SPSS for Windows version 6.0.1 statistical software package (SPSS Inc., Chicago, IL) was used to analyze the data. A three-factor repeated-measures analysis of variance was used to determine if any differences existed between Tester 1 and Tester 2 for joint position sense. The dependent variable was joint position sense, measured in degrees of error. The independent variables included tester (Tester 1 vs. Tester 2$)$, mode (active vs. passive), and position $\left(5^{\circ}\right.$ from maximum inversion vs. $15^{\circ}$ inversion vs. $0^{\circ}$ neutral vs. $10^{\circ}$ eversion). If a significant $\mathrm{F}$ ratio ( $\mathrm{p}<$ .05) was obtained, a Tukey post hoc test was utilized to determine where the differences occurred. In addition, separate repeated-measures analyses of variance were performed on each test condition. Reliability was estimated via intraclass correlation coefficients (ICC), while standard errors of measurement (SEM) were used to estimate the accuracy of measurement (4). Separate ICCs were derived from the existing separate repeated-measures ANOVA outputs using Shrout and Fleiss's (25) formula (2,k). SEMs were computed by multiplying the standard deviation of the mean degree of error score for each testing condition by the square root of 1 minus the corresponding ICC.

\section{RESULTS}

The means and standard deviations for active and passive joint position sense (JPS) according to testers are shown in Table 1. The three-factor repeated- measures ANOVA revealed no significant main effects for tester, $\mathrm{F}(1,19)=0.18, \mathrm{p}=.674$; mode, $\mathrm{F}(1,19)=1.16, \mathrm{p}=.295$; or position, $\mathrm{F}(3,57)=0.51, \mathrm{p}=.674$. There was a significant interaction for mode by position, $\mathrm{F}(3$, $57)=$ testing speed. The subject was positioned supine on a modified examination table with the lower leg parallel to the floor, resting on a 10-in. high rectangular support (25 in. wide and 14 in. long) (Figure 1). This setup enabled proper placement of the subject's bare foot into the Kin-Com II ankle inversion/eversion apparatus with the ankle plantar flexed $25^{\circ}$. The ankle to be tested was randomly selected. The heel support of the apparatus was lined with $1 / 2 \mathrm{in}$. foam, and the subject's midfoot and forefoot were wrapped approximately three times with $1 / 8 \mathrm{in}$. Elastifoam wrap (low-density elastic foam) to decrease external sensory stimulation. After the subject's foot was properly aligned with the axis of the isokinetic dynamometer according to manufacturer's guidelines, stabilization was provided by placing Velcro straps around the proximal tibiofibular joint and forefoot (Figure 1). Subjects were required to close their eyes during the testing procedures to eliminate visual feedback.

Active and passive joint repositioning occurred at the following four test positions: $10^{\circ}$ eversion, $0^{\circ}$ subtalar neutral, $15^{\circ}$ inversion, and $5^{\circ}$ from maximum inversion. Subtalar neutral served as the $0^{\circ}$ neutral position. This position was individually determined by palpating for equal distribution of the head of the talus bone medially and laterally while passively inverting and everting the foot (16). The $5^{\circ}$ from maximum inversion test position was established by having the subject actively invert the foot maximally. This position was visually displayed in degrees by

the internal goniometer of the Kin-Com II. Testing order was counterbalanced according to mode 
(active/passive) and test position $\left(10^{\circ}\right.$ eversion $/ 0^{\circ}$ subtalar neutral, $15^{\circ}$ inversion $/ 5^{\circ}$ from maximum inversion). The preselected testing order and side of body tested during the initial evaluation were replicated during the second evaluation.

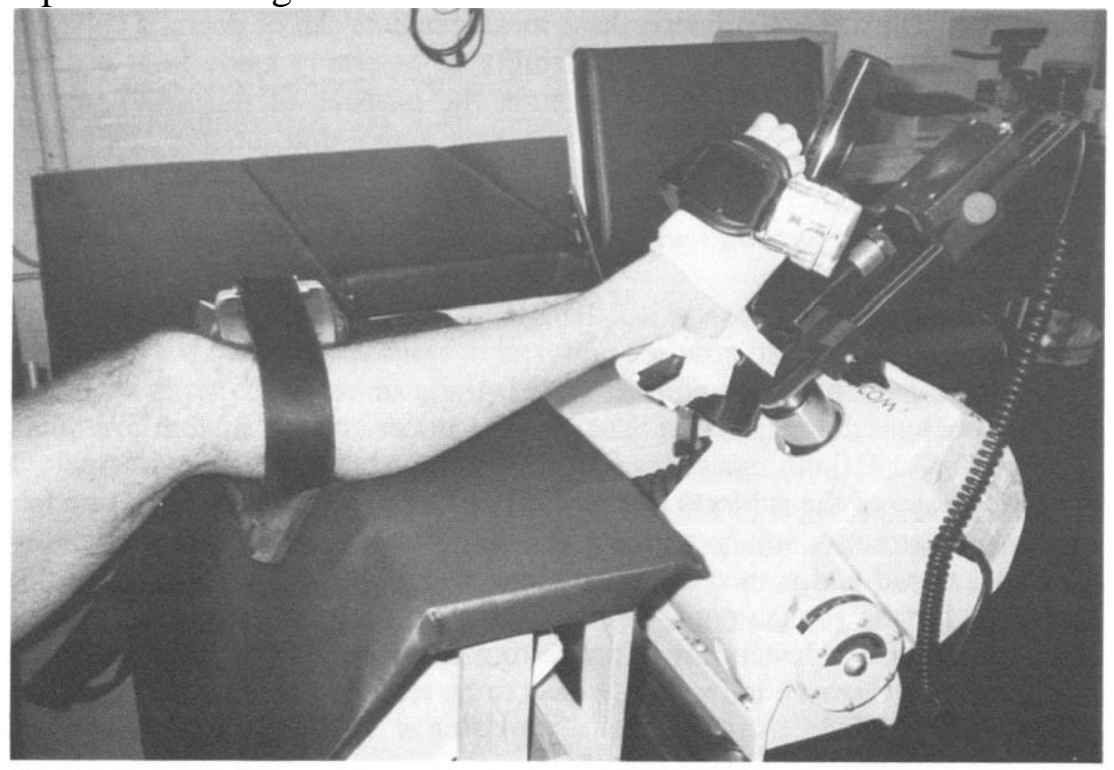

Figure 1 - Positioning of subject on the Kin-Com II isokinetic dynamometer for ankle joint position sense testing.

Prior to being tested, each subject received a practice session followed by a $30-\mathrm{s}$ rest period. The testing began by the investigator passively moving the subject's foot through its entire inversion and eversion range of motion. The foot was then passively moved into the preselected test position where it was held for $15 \mathrm{~s}$. Once at the test position, the subject concentrated on the test angle. The foot was then passively moved back to the starting position, which was end range of maximal inversion for the $0^{\circ}$ subtalar neutral and $10^{\circ}$ eversion test positions, and end range of maximal eversion for the $15^{\circ}$ inversion and $5^{\circ}$ from maximum inversion test positions. Once the starting position had been reached, the investigator proceeded to passively move the subject's foot back in the direction of the test position. When the subjects felt that their foot position replicated that of the testing angle they performed a quick contraction of the foot in the direction opposite the passive motion. This contraction was then recorded as a spike on the forcevelocity curve output on the Kin-Com II computer screen. From this spike on the curve, the investigator could then extrapolate the initial angle of movement that corresponded to subject's repositioned joint angle. Joint position sense measurements were recorded in degrees of error from the test position. Two trials were performed for each sequence. The absolute values of each error score were then averaged together and recorded for analysis. Active joint position sense testing occurred under an identical protocol except that the subject actively moved his or her foot to replicate the test position after being moved to the start position.

\section{Statistical Analysis}

The SPSS for Windows version 6.0.1 statistical software package (SPSS Inc., Chicago, IL) was used to analyze the data. A three-factor repeated-measures analysis of variance was used to determine if any differences existed between Tester 1 and Tester 2 for joint position sense. The dependent variable was joint position sense, measured in degrees of error. The independent variables included tester (Tester 1 vs. Tester 2 ), mode (active vs. passive), and position ( $5^{\circ}$ from 
maximum inversion vs. $15^{\circ}$ inversion vs. $0^{\circ}$ neutral vs. $10^{\circ}$ eversion). If a significant $\mathrm{F}$ ratio ( $\mathrm{p}<$ $.05)$ was obtained, a Tukey post hoc test was utilized to determine where the differences occurred. In addition, separate repeated-measures analyses of variance were performed on each test condition. Reliability was estimated via intraclass correlation coefficients (ICC), while standard errors of measurement (SEM) were used to estimate the accuracy of measurement (4). Separate ICCs were derived from the existing separate repeated-measures ANOVA outputs using Shrout and Fleiss's (25) formula (2,k). SEMs were computed by multiplying the standard deviation of the mean degree of error score for each testing condition by the square root of 1 minus the corresponding ICC.

\section{RESULTS}

The means and standard deviations for active and passive joint position sense (JPS) according to testers are shown in Table 1. The three-factor repeated- measures ANOVA revealed no significant main effects for tester, $\mathrm{F}(1,19)=0.18, \mathrm{p}=.674$; mode, $\mathrm{F}(1,19)=1.16, \mathrm{p}=.295$; or position, $\mathrm{F}(3,57)=0.51, \mathrm{p}=.674$. There was a significant interaction for mode by position, $\mathrm{F}(3$, 57) $=$ 
Table 1 Means and Standard Deviations for Joint Position Sense Measurements, in Degrees of Error, for Tester 1 and Tester 2

\begin{tabular}{llllll}
\hline \multirow{2}{*}{ Position } & & \multicolumn{2}{c}{ Tester 1 } & \multicolumn{2}{c}{ Tester 2 } \\
& Mode & $M$ & $S D$ & $M$ & $S D$ \\
\hline \multirow{2}{*}{$5^{\circ}$ from max inv } & Passive & 4.50 & 5.41 & 4.55 & 3.00 \\
& Active & 8.55 & 6.71 & 7.58 & 4.27 \\
$15^{\circ}$ inversion & Passive & 6.60 & 5.02 & 5.63 & 4.04 \\
& Active & 6.95 & 5.09 & 5.65 & 4.25 \\
$0^{\circ}$ neutral & Passive & 5.20 & 3.46 & 6.68 & 5.86 \\
& Active & 5.50 & 4.73 & 6.55 & 4.20 \\
$10^{\circ}$ eversion & Passive & 8.00 & 6.23 & 6.60 & 5.91 \\
& Active & 6.80 & 4.23 & 6.62 & 4.86 \\
& & & & & \\
\hline
\end{tabular}

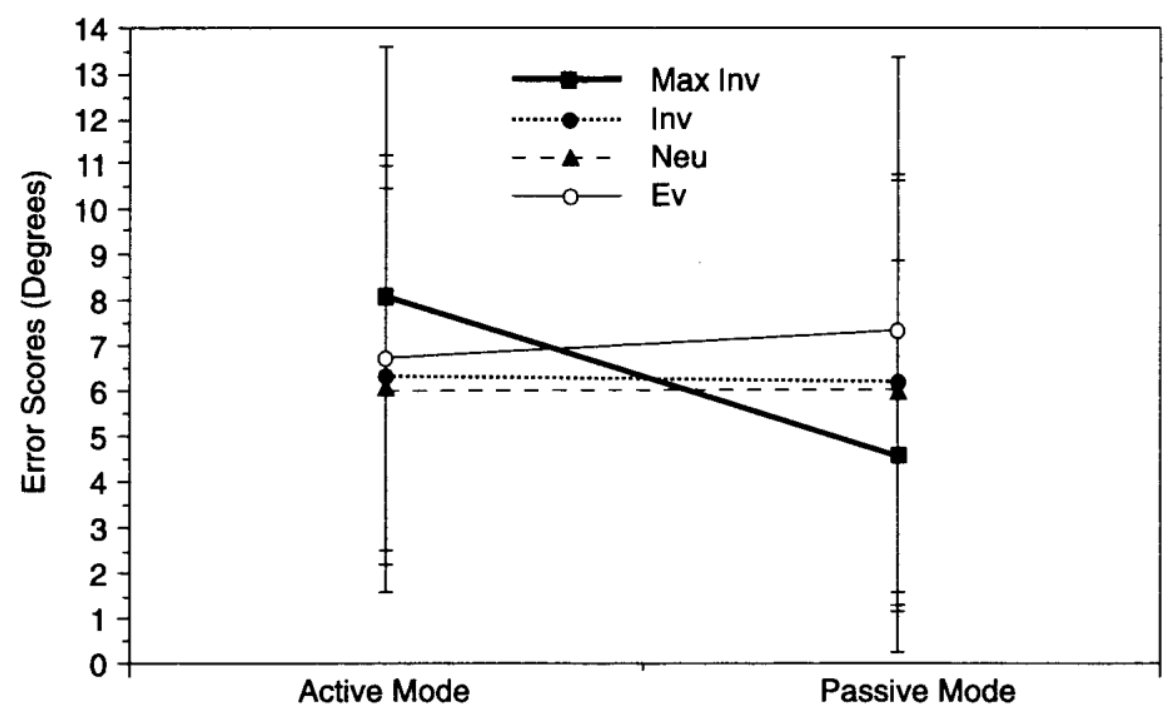

Figure 2-Graph of significant interaction for joint position sense error scores (degrees) between the active and passive modes at the $5^{\circ}$ from maximum inversion position.

4.57, $\mathrm{p}=.006$ (Figure 2). The Tukey post hoc analysis indicated that the active mode yielded higher joint position error scores than did the passive mode in the $5^{\circ}$ from maximum inversion position $(\mathrm{p}<.05)$.

The sampled population means and standard deviations, intraclass correlation coefficients (ICC), and standard errors of measurement (SEM) are presented in Table 2. The mean joint position error scores ranged from 6.03 to $8.06^{\circ}$ for active JPS and 4.53 to $7.30^{\circ}$ for passive JPS. The intraclass correlation coefficients (ICC 2,k) ranged from .03 to .51 and from .08 to .87 for both active and passive

Table 2 Sample Population Means and Standard Deviations, Intraclass Correlation Coefficients (ICC), and Standard Error of Measurements (SEM) for Joint Position Sense Measurements (degrees) 


\begin{tabular}{llllll} 
Position & Mode & $M$ & $S D$ & ICC & SEM \\
\hline \multirow{2}{*}{$5^{\circ}$ from max inv } & Passive & 4.53 & 4.32 & .08 & 4.14 \\
& Active & 8.06 & 5.56 & .51 & 3.89 \\
$15^{\circ}$ inversion & Passive & 6.11 & 4.52 & .87 & 1.63 \\
& Active & 6.30 & 4.68 & .03 & 4.61 \\
$0^{\circ}$ neutral & Passive & 5.94 & 4.86 & .14 & 4.51 \\
& Active & 6.03 & 4.45 & .12 & 4.17 \\
$10^{\circ}$ eversion & Passive & 7.30 & 6.04 & .70 & 3.31 \\
& Active & 6.71 & 4.50 & .50 & 3.18
\end{tabular}

Table 3 Between-Subjects Mean Square (BMS), Error Mean Square (EMS), and Trial Mean Square (TMS) for Calculating Intraclass Correlation Coefficients (ICC)

\begin{tabular}{llrrrr} 
Position & Mode & BMS & EMS & TMS & ICC \\
\hline \multirow{2}{*}{$5^{\circ}$ from max inv } & Passive & 18.43 & 19.83 & 0.02 & .08 \\
& Active & 42.21 & 20.79 & 9.03 & .51 \\
$15^{\circ}$ inversion & Passive & 36.90 & 4.61 & 9.51 & .87 \\
& Active & 21.72 & 22.31 & 16.90 & .03 \\
$0^{\circ}$ neutral & Passive & 25.23 & 21.70 & 28.06 & .14 \\
& Active & 21.26 & 18.79 & 11.03 & .12 \\
$10^{\circ}$ eversion & Passive & 56.68 & 17.07 & 19.60 & .70 \\
& Active & 27.39 & 14.10 & 0.31 & .50
\end{tabular}

JPS, respectively. Corresponding standard errors of measurement ranged from 3.18 to $4.61^{\circ}$ actively and 1.63 to $4.51^{\circ}$ passively. The between-subjects mean square (BMS), error mean square (EMS), and trial mean square (TMS) values for calculating the ICCs are listed in Table 3.

\section{DISCUSSION}

The primary findings of our study indicated that a wide range of reliability estimates exist for active and passive testing conditions and that passive repositioning elicited significantly smaller error scores than active repositioning at the $5^{\circ}$ from maximum inversion position. Several studies have produced results suggesting that passive joint position sense is significantly better (less calculated error) than active joint position sense $(2,13,17)$. In a study investigating the effect of a 6-week balance and coordination training program on proprioception of functionally unstable ankles, Bernier's (2) control, sham, and treatment groups all exhibited significantly higher error scores when tested under the active mode as compared to the passive mode. In accordance with Bernier (2), Kaminski and Perrin (17) found that regardless of the knee brace condition, their subjects produced significantly less error during passive repositioning of knee joint angles as compared to active repositioning.

Both Bernier (2) and Kaminski and Perrin (17) supported their claims based upon a previous study that examined the effects of chronic lateral ankle sprains on active and passive judgments of joint position (13). This study, conducted by Gross (13), indicated that passive judgments of ankle joint position were significantly better than active judgments of joint position in individuals free from ankle injuries. Gross (13) accounted for the increased error score associated with active repositioning via the theory that while joint receptors are more likely responsible for 
sensing actual joint position, muscle receptors are more reliant for detecting joint movement. Thus, Gross (13) hypothesized that the increased muscle afferent and efferent input during active repositioning was actually detrimental to sensing joint position because of possible processing errors. In addition, Bernier (2) suggested that the slow speed at which joint position sense was measured in her study $(5 \%$, which was also used in the present study) may have discriminated against muscle proprioceptors that have been believed to best sense rapid changes in joint movement.

Contrary to these findings, our results did not reveal a significant main effect for mode, yet there was a significant mode by position interaction. The Tukey post hoc analysis indicated that passive position sense error scores $\left(4.53 \pm 4.32^{\circ}\right)$ were significantly smaller than those obtained during the active mode $\left(8.06 \pm 5.57^{\circ}\right)$ at the $5^{\circ}$ from maximum inversion test position. This finding coincides with that of Bernier (2), who observed mean joint position sense scores of 4.53 $\pm 3.2^{\circ}$ and $7.85 \pm 5.7^{\circ}$ for the passive and the active modes, respectively, in the $5^{\circ}$ from maximum inversion position. Somewhat contradictory results were previously established by Glencross and Thornton (11), who reported a positive linear trend between the amount of passive repositioning error produced and the degree of the test angle. They found that the closer the test angle was to the terminal degrees of either plantar flexion or dorsiflexion, the greater the amount of passive repositioning error recorded (11).

In both the present study and the one conducted by Bernier (2), mean joint position sense scores were lowest when joints were tested passively and highest when tested actively in the $5^{\circ}$ from maximum inversion position. An additional factor that may account for the passive repositioning being less than the active at the $5^{\circ}$ from maximum inversion position may be related to the test protocol used in this and other studies $(2,11,17)$. That is, active repositioning following passive positioning may confound differing sensory inputs to the CNS. Future research should compare the influence of passive versus active positioning on active repositioning.

Intraclass correlations make it possible to distinguish between score variances that are due to differences between subjects as opposed to those due to measurement error or changes in scores over time. In general, an ICC is a ratio of subject differences to the total score variance (including error variance) (27). The numerator of the formula represents the amount of variance accounted for between subjects on a particular measurement. If there is a relatively small between-subject variation, ICCs will tend to be low. Therefore, scores among a group with little variance, although consistent from one time to the next or one tester to another, may produce low ICCs (4). In our study, the four lowest ICCs were associated with the smallest between-subject variances (Table 3 ).

The intertester reliability values of this study for active and passive ankle joint position sense testing varied considerably. The ICCs ranged from a low of $.03\left(\mathrm{SEM}=4.61^{\circ}\right)$ for the active $15^{\circ}$ inversion position to a high of $.87\left(\mathrm{SEM}=1.63^{\circ}\right)$ for the passive $15^{\circ}$ inversion position. Subjects' scores at this test position for the passive mode were much more diversified and consistent than the scores yielded during the active mode. This finding clearly supports Gross's (13) hypothesis regarding the function of joint and muscle mechanoreceptors as well as Bernier's (2) in reference to the slow testing speed. 
Reliability estimates for both active $\left(\mathrm{ICC}=.12, \mathrm{SEM}=4.17^{\circ}\right)$ and passive $(\mathrm{ICC}=.14, \mathrm{SEM}=$ $4.51^{\circ}$ ) joint position sense measurements at the subtalar neutral position were very low, and standard errors of measurement for these conditions were relatively high. Between-subject variance was low in these conditions, and there was little consistency in subjects' scores between testers.

Another factor could be the difficulty in establishing a consistent subtalar neutral position between testers $(5,24,26)$, which would inevitably lead to the assessment of different joint angles by inquiring investigators. Joint position sense measurements recorded at the $10^{\circ}$ eversion position produced a low to moderate reliability estimate $\left(\mathrm{ICC}=.70, \mathrm{SEM}=3.31^{\circ}\right)$ for the passive mode and a poor reliability estimate $\left(\mathrm{ICC}=.50, \mathrm{SEM}=3.18^{\circ}\right)$ for the active mode. Between- subject variance was highest in the passive mode, and there was a low consistency in scores from one tester to the next for both modes of testing. This also was true for the active $\left(\mathrm{ICC}=.51, \mathrm{SEM}=3.89^{\circ}\right)$ and passive $\left(\mathrm{ICC}=.08, \mathrm{SEM}=4.14^{\circ}\right)$ modes at the $5^{\circ}$ from maximum inversion test position. Between-subject variance was relatively high in the active mode. In the passive mode, between- subject variance was low and subject scores were inconsistent from one time to the next. More specifically, some subject error scores went up while others went down.

Unfortunately, to the best of our knowledge, there have been no reported reliability estimates or standard errors of measurement for testing ankle joint position sense. Attempting to explain the wide range of differences in the reliability estimates presents a challenge since no real distinctive pattern is portrayed. We have suggested a number of reasons why some of the correlations are so low. Several potential causes for the fluctuating range of reliability may lie within the actual experimental protocol used in this study. The assessment of the subtalar neutral joint position particularly warrants investigation. Intertester reliabilities for determining the subtalar neutral joint position in the open kinetic chain have been documented to range from ICCs $(1,1)$ equivalent to $.00\left(\mathrm{SEM}=2.51^{\circ}\right)(24)$ and $.25(5)$ for the inexperienced tester to an ICC $(3,1)$ equivalent to .60 (26) for the experienced tester. Reliabilities such as these have prompted researchers to encourage clinicians to practice this measurement technique repeatedly before assessing lower extremity dysfunctions (24). Since the exact techniques used to determine subtalar neutral and the experience of the investigators were slightly different between the cited and present studies, it is difficult to render a direct comparison. But the general concern regarding intertester reliability of determining the subtalar neutral position remains noteworthy and cannot be overlooked regarding the outcome of our study.

Another area of concern was the placement of the subject's foot in the Kin-Com II ankle inversion/eversion apparatus. An attempt was made to decrease external sensory stimulation by placing a foam substance around each subject's midfoot and forefoot and by also padding the heel support of the apparatus. Nevertheless, subjects reported isolated instances where part of their foot or ankle may have contacted part of the apparatus or experienced some external exerted pressure, especially during the active joint repositioning sequence. A second concern regarding the foam padding involved a compression effect exhibited between the subject's foot and the ankle apparatus during the active mode. It was apparent during the active tests that the subject's foot compressed the foam, perhaps causing the subject's foot to prematurely obtain the test angle in reference to the joint angle that was being recorded by the internal goniometer of the isokinetic testing device. This reported discrepancy in the actual and machine joint angles 
resulted in some subjects strategically overshooting what they believed to be the test position to compensate for this compression effect. This undoubtedly affected the reliability of these measures. It seems apparent that the foam padding used in our study may have caused more problems than it solved. Perhaps excluding the foam padding and testing subjects with their shoes on would help increase the stability of the foot in the testing apparatus (18) and help decrease the effect of external sensory stimulation.

In summary, this investigation did not find a high intertester reliability for active or passive ankle joint position sense when employing the specific methods described. Consumers of research should consider the methods used for determining ankle joint position sense in the interpretation of a study's findings. Additional research is needed to enhance the reliable assessment of ankle joint position sense.

\section{REFERENCES}

1. Andersen, S.B., D.M. Terwilliger, and C.R. Denegar. Comparison of open versus closed kinetic chain test positions for measuring joint position sense. J. Sport Rehabil. 4:165-171, 1995.

2. Bernier, J.N. Effect of coordination training on proprioception of the functionally unstable ankle. Unpublished doctoral dissertation, University of Virginia, Charlottes ville, 1995.

3. Brooks, S.C., B.T. Potter, and J.B. Rainey. Treatment for partial tears of the lateral ligaments of the ankle: A prospective trial. Br. Med. J. 282:606-607, 1981.

4. Denegar, C.R., and D.W. Ball. Assessing reliability and precision of measurement: An introduction to intraclass correlation and standard error of measurement. J. Sport Rehabil. 2:35-42, 1993.

5. Elveru, R.A., J.M. Rothstein, and R.L. Lamb. Goniometric reliability in a clinical setting: Subtalar and ankle joint measurements. Phys. Ther. 65:672-677, 1988.

6. Freeman, M.R. Treatment of ruptures of the lateral ligament of the ankle. J. Bone Joint Surg. (Br.) 47:661-668, 1965.

7. Freeman, M.R. Instability of the foot after injuries to the lateral ligament of the ankle. $J$. Bone Joint Surg. (Br.) 47:669-677, 1965.

8. Freeman, M.R., M.E. Dean, and L.F. Hanham. The etiology and prevention of functional instability of the foot. J. Bone Joint Surg. (Br.) 47:678-685, 1965.

9. Garn, S.N., and R.A. Newton. Kinesthetic awareness in subjects with multiple ankle sprains. Phys. Ther. 68:1667-1671, 1988.

10. Garrick, J.G. The frequency of injury, mechanism of injury, and epidemiology of ankle sprains. Am. J. Sports Med. 5:241-242, 1977.

11. Glencross, D., and E. Thomton. Position sense following joint injury. J. Sport Med. Phys. Fit. 21:23-27, 1981.

12. Grigg, P. Peripheral neural mechanisms in proprioception. J. Sport Rehabil. 3:2-17, 1994.

13. Gross, M.T. Effects of recurrent lateral ankle sprains on active and passive judgments of joint position. Phys. Ther. 67:1505-1509, 1987.

14. Hertel, J.N., K.M. Guskiewicz, D.M. Kahler, and D.H. Perrin. Effect of lateral ankle joint anesthesia on joint position sense, postural sway and center of balance. (in review). 
15. Irrgang, J.J., S.L. Whitney, and E.D. Cox. Balance and proprioceptive training for rehabilitation of the lower extremity. J. Sport \&Rehabil. 3:68-83, 1994.

16. James, S.L. Chondromalacia of the patella in the adolescent. In The Injured Adolescent Knee, J.C. Kennedy (Ed.). Baltimore: Williams \& Wilkins, 1979, pp. 214-228.

17. Kaminski, T.W., and D.H. Perin. Effect of prophylactic knee bracing on balance and joint position sense. (in review).

18. Kaminski, T.W., D.H. Perrin, C.G. Mattacola, J.E. Szczerba, and J.N. Bernier. The reliability and validity of ankle inversion and eversion torque measurements from the Kin Com II isokinetic dynamometer. J. Sport Rehabil. 4:210-218, 1995.

19. Konradsen, L., J.B. Ravn, and A.I. Sorensen. Proprioception at the ankle: The effect of anaesthetic blockade of ligament receptors. J. Bone Joint Surg. (Br.) 75:433-436, 1993.

20. LaRiviere, J., and L.R. Osternig. The effect of ice immersion on joint position sense. $J$. Sport Rehabil. 3:58-67, 1994.

21. Lentell, G.L., B. Baas, D. Lopez, L. McGuire, M. Sarrels, and P. Snyder. The contributions of proprioceptive deficits, muscle function, and anatomic laxity to functional instability of the ankle. J. Orthop. Sport Phys. Ther. 21:206-215, 1995.

22. Lentell, G.L., L.L. Katzman, and M.R. Walters. The relationship between muscle function and ankle stability. J. Orthop. Sport Phys. Ther. 11:605-611, 1990.

23. Lephart, S.M., M.S. Kocher, F.H. Fu, and C.D. Hamer. Proprioception following anterior cruciate ligament reconstruction. J. Sport Rehabil. 1:188-196, 1992.

24. Picciano, A.M., M.S. Rowlands, and T. Worrell. Reliability of open and closed kinetic chain subtalar joint neutral positions and navicular drop test. J. Orthop. Sport Phys. Ther. 18:553-558, 1993.

25. Shrout, P.E., and J.L. Fleiss. Intraclass correlations: Uses in assessing rater reliability. Psycho!. Bull. 86:420-428, 1979.

26. Smith-Oricchio, K., and B.A. Harris. Interrater reliability of subtalar neutral, calcaneal inversion and eversion. J. Orthop. Sport Phys. Ther. 12:10-15, 1990.

27. Stratford, P. Reliability: Consistency or differentiating among subjects? Phys. Ther. 69:299300, 1989.

28. Tropp, H. Pronator muscle weakness in functional instability of the ankle joint. Int. J. Sport Med. 7:291-294, 1986. 